\title{
Application of the quadrature-difference method for solving Fredholm integro- differential equations
}

\begin{abstract}
Application of the quadrature-difference method for solving Fredholm integro-differential equations (FIDEs) is presented in this paper. The FIDEs will be discretized by using the combinations of Simpsons quadrature rule with finite difference. The method converts the FIDEs to a matrix equation which corresponds to a system of linear algebraic equations. The formulation and the implementation of the proposed method are also presented. Several numerical examples are presented to illustrate the performance of the proposed method.
\end{abstract}

Keyword: Finite difference; Fredholm integro-differential equations; Gauss elimination; Quadrature rule; Simpsons rule 